\title{
RIDUZIONE ISOSTATICA DELLE DETERMINAZIONI (;RAYIVETRICHE ESEGUITE SUI COLLI ALBANI
}

\author{
Carmelo douilana
}

Vei pas-ali ami lo srisente ha eseruilo, sui Colli Albani e nei

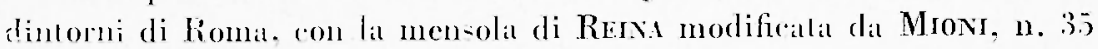
-lazioni per la delerminazione relativa di gravila. lulle collegate al founto di accelerazione gravimetrica notal di Roma (Facolla d'IngeEnelia a S. Pietro in Vincoli) $(y=980.36 \pi+0.003)$.

I risulati fonali di que-le oservazioni, ridolle secondo l’ipolesi di Fus, o le relatire anomalie già pubblicale in nole precedenti (') -ono riporlate in lab. I.

In seruilo, poiche era nella lendenzil degli -renziati ilaliani di

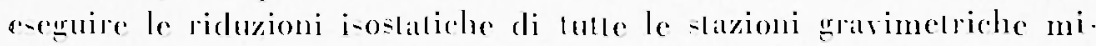

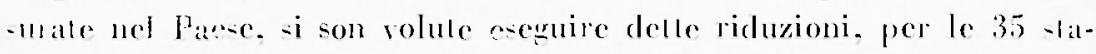
zimi anzicleile.

In fue-to lavoro rendiano note appunto le anomalie ollenule - ia dopo arer apportate le correzioni sceondo Harfond-Bowls (Prall),

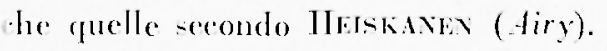

La profondila di compensizione per la riduzione secondo Harono -i i. firala a km 96, e, per la riduzione secondo Helshaver, a km 60. le riduzioni -ono sate alleolate ulilizzando le "Tarole fondamentali por la riduzione dei valori ossertuli della gratila n di G. Cassixs, "'. Dore e S. Batentay e adoltando lo shema di suddivisione in - rompartimenti "ripollalo in delle lavole.

La delerminazione delle quole dei compartimenli i is lalla, nelle immediate vicinanze delle stazioni gravimetriche, su carte lopogratfiche a grande scala, $c$, man mano else ci si allonlanara, su carte 1:25.0100 dell'T.G.M. (fino alla zona G inclusa); 1:100.000 dell'T.G.M. (fino alla zona I, inclusa); 1:500.000 degli Alanti: André e Stieler (dalla zona $\mathrm{I}_{2}$ alla zona $\mathrm{O}_{1}$ ): carla $1: 3.000 .000$ dell'Allanle Andree (dalla zona (), alla 16); rarla $1: 12.000 .000$ per l'Europa o $1: 10.000 .000$

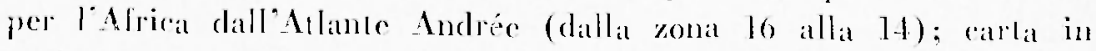
proiczione equivalente dellinlera superfeie terestre per le zone reLimti. 


\section{TABella I}

\begin{tabular}{|c|c|c|c|c|c|c|c|}
\hline $\begin{array}{c}\text { Stazioni } \\
\mathrm{S}\end{array}$ & $\begin{array}{l}\text { Lation- } \\
\text { diue }\end{array}$ & Long & ituline & $\mid \begin{array}{c}A \text { ltezza } \\
H \\
\text { del } \\
\text { punto } \\
\mathrm{S}\end{array}$ & $\begin{array}{c}\text { Gravitit } \\
\text { ridotta } \\
\text { secondo } \\
\text { FiYE } \\
\text { g' }\end{array}$ & $\begin{array}{c}\text { (Yravità } \\
\text { normale } \\
(1930) \\
Y\end{array}$ & $\begin{array}{l}\text { Anomg - } \\
\text { lio } \\
\mathbf{g}^{\prime}-\because\end{array}$ \\
\hline Roceat di P'apal & $410.15,9$ & 0015.5 & E.MI.M. & $759 \mathrm{~m}$ & $\begin{array}{c}\text { gall } \\
980.132\end{array}$ & $\begin{array}{c}\text { gall } \\
\text { yo(1).338 }\end{array}$ & $\begin{array}{c}\text { gal } \\
+\quad 0.091\end{array}$ \\
\hline Montr Caro & $" 45.1$ & 15.5 & $n$ & $951 \%$ & 980.131 & 980.337 & +0.0001 \\
\hline Ariecia & $\Rightarrow 13,1$ & " 13.1 & " & $1013 n$ & 980,398 & 580.3331 & +0.0601 \\
\hline Caaklel Gallollollo & $\Rightarrow 13,8$ & 711.6 & ) & 1350 & 980.1211 & 980.2366 & $\therefore 0.1181$ \\
\hline Marino & $\Rightarrow 16.1$ & $" 12.5$ & $n$ & 3590 & 980.115 & 980.338 & $\div 0.07 \%$ \\
\hline liratsuali & $n 48.6$ & ") $13,5,5$ & " & $32 \because "$ & 980.1013 & 980.312 & +0.1661 \\
\hline Cianpino & 1) 17.9 & 1) 08.1 & ") & $110 \ldots$ & 980.120 & 980.311 & +0.0170 \\
\hline Eratlorerhie & 116.0 & $" 119.3$ & $1)$ & 18011 & 980.1116 & 980.338 & +11.068 \\
\hline$V_{t^{2}} m i$ & 1113.1 & " 15.1 & m & 5213 & 980.116 & 980.33 .1 & +0.082 \\
\hline Velletri & $\Rightarrow 11.3$ & $" 19.6$ & $"$ & $371 \mathrm{~m}$ & 980.390 & 980.331 & +0.068 \\
\hline Monteromulatri & $" 13.3$ & 1) 17.0 & 1) & $5.30)$ & 980,120 & 980.312 & $\div 0.078$ \\
\hline Rocea Prioria & $\Rightarrow-17,6$ & $\Rightarrow 18.7$ & ) & $768 n$ & 980.118 & 980.3410 & $\because 11.1178$ \\
\hline Cilpo di Bove & $\Rightarrow 50.6$ & 1105.9 & $"$ & $71 »$ & 900.287 & 930.345 & +11.011 .1 \\
\hline Sic. M. Agraria & $" 50.2$ & $n 03.7$ & $n$ & $51 "$ & 980.371 & 980.345 & +11.1129 \\
\hline IV Mirrlio & $" 50.1$ & $\Rightarrow 0.1 .3$ & 1) & $55 \mathrm{D}$ & 980.375 & 980.345 & +0.030 \\
\hline oalstel di Leval & $" 45.8$ & 115.3 & ") & $102 "$ & 900.361 & 980.338 & +0.026 \\
\hline Sc. D. C. Batsi & 348,3 & 1702.2 & i) & $50 \%$ & 980.362 & 980.311 & $-1 \cdot 0.021$ \\
\hline Mandriolal & $" 15.1$ & $n 02.7$ & 11 & 710 & 980.378 & 980.338 & -0.010 \\
\hline Lanuvio & 1) $10, \cdot 1$ & $" 11 . \vec{\imath}$ & $"$ & 32010 & 080.375 & 980.330 & +0.01 .17 \\
\hline Vistri (0.M.l.1 & " 51.5 & $\Rightarrow 01.2$ & ") & $18 "$ & 900.373 & 4830.317 & $4-0.026$ \\
\hline Payona & $11+1.0$ & $" 09.7$ & $"$ & $190 "$ & 980.388 & 980.335 & +0.053 \\
\hline Corerhina & $n-41.5$ & $" 11.7$ & $"$ & $216 \quad$ & 930.360 & 980.3 .31 & -11.037 \\
\hline$\therefore$ Cinarou & ;1 19,3 & $\Rightarrow 21.11$ & $b$ & $315 n$ & 980.396 & 980.313 & +11.053 \\
\hline Pantano & " 52,3 & "15.6 & 3 & $56 \%$ & $900.37 \%$ & 980.3 .17 & -0.0130 \\
\hline Turre Vuval & 1751.6 & $" 09.9$ & $n$ & $62 n$ & 980.370 & 9811.347 & +0.023 \\
\hline Gisterna & $" 35,5$ & $" 2 \cdot 2 \cdot \overline{7}$ & " & $76 n$ & $\sin 80.390$ & 980.322 & $+11.06 \overline{7}$ \\
\hline Cori & $\geqslant 38.5$ & $" 27 . \overline{7}$ & $n$ & 395 & 90.11 .47 & 980.327 & --0.120 \\
\hline Giulianello & 111.1 & $n 25.4$ & 1) & $225 "$ & 980.396 & 980.331 & $\therefore 0.06 .5$ \\
\hline l.ariano & 1) -13.6 & 123.0 & 》 & 3.540 & 380.113 & 980.335 & +0.1178 \\
\hline Valmonlone & 16.6 & 128.0 & $\because$ & 3033 & 980.386 & 980.339 & +0.017 \\
\hline Pallestrina & $" 50.3$ & y) 26.3 & ") & $16.5 \mathrm{~B}$ & 980.120 & $980.3-45$ & -0.1175 \\
\hline (;illicano & $1) 52.3$ & $\Rightarrow 22.0$ & ") & $211 \mathrm{D}$ & 980.395 & $9811,3.18$ & +0.0 .47 \\
\hline Ten. Granararedio & $n 5 \overline{5} .1$ & 112.2 & 1) & 511 & 980.391 & 980.352 & -+0.0139 \\
\hline I.ungleezzal & $" 55.5$ & $\Rightarrow 12.9$ & $"$ & $41 \%$ & 080.381 & 980.352 & +0.1129 \\
\hline Sullocamini & 156.3 & 1) 10.3 & $"$ & $13 n$ & 980,335 & 980.35 .1 & $\therefore 0.0131$ \\
\hline
\end{tabular}


La dem-ila media della cro-ta lerrestre per le riduzioni serondo

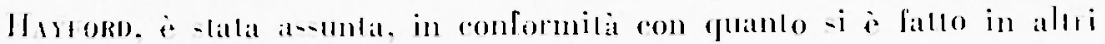
lavori dello -lewo genere, uguale al 2.67 : quella dei mari l.113.

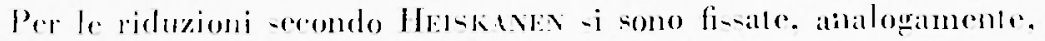
per il Sial la den-ilà di 2,67 a per il Sima 3.27.

I risulati ollenuli sono riporlati nella seguente labella $I I$.

Dit deltat labellat rileviamo the $\mathrm{i}$ valori ridolti seguendo il pro-

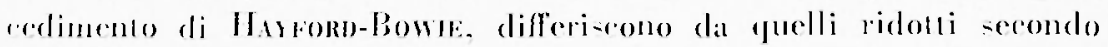

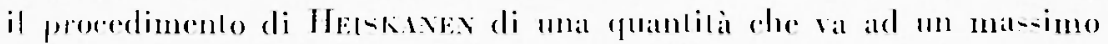

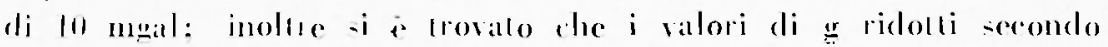

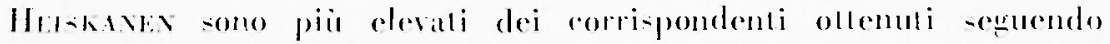
Hayorm. Na a rio non altribuiamo alem signifiealo.

b-emiano intine the i valori di $\stackrel{2}{=}$ ridolli secondo le ipole-i

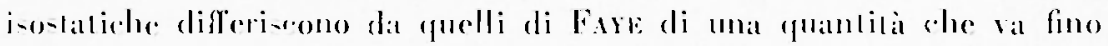

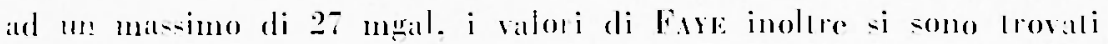
-uperiori dei corri-pondenti ridolli sceondo le ipoteri isostalliche.

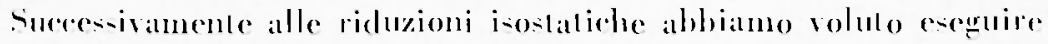
anche la riduzione lobale sereondo Botacker per le ragioni seguenti. Aello suclio intensivo di regioni ristrelle. quando a si propone di

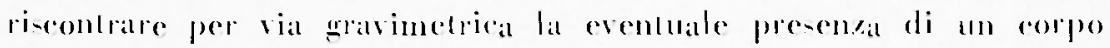

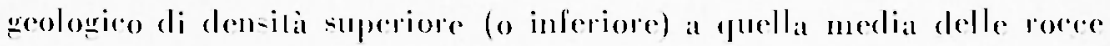
rireosanti interessi grandemente lener ronlo, wià in sede di correzione, della distribuzone effelliva della densila in modo da " i-olare"

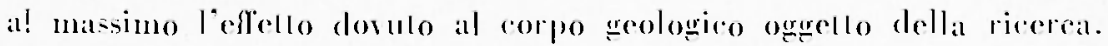
Ora esendo gli effelli delle parli nole minimizzali o ammullali dalle

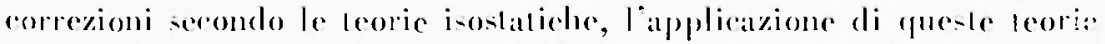
non appare senzaltro giuslifieabile in rasi cone quello in calme.

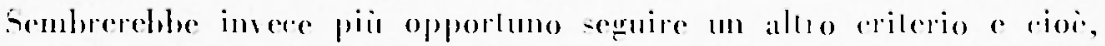
trovalo per ogni pumlo di stazome il valore della pravila, calcolate il valore defl'anomalia delraendo da eno il valore della gravila de ra quello -leso punlo di stazione i misurerebbe nel raso irleale di a-enza del rorpo di dem-ila diversal ferme restando lutle le altre cal-

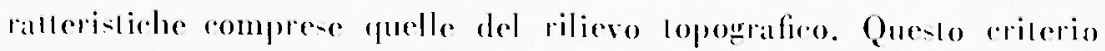
ronduce in defonitiva ad apporiare alle mi-ure della gravila la ridu-

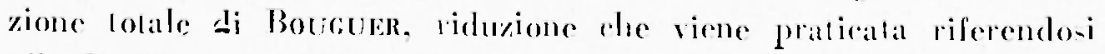
alla den-ila media delle roce circostanli, sia per il caleolo della pia- ha rhe pee fuello della correxione topografieal.

Vella lahella III sono tali riportali i valori ollemuli apporlando alle mi-nre delle 35 tazioni la riduzione loble di Bougutar arendo 
Tabelata II

Anomalic secondo Hayford e secondo Heislianen

\begin{tabular}{|c|c|c|c|c|}
\hline \multirow{2}{*}{$\begin{array}{l}\text { Stazioni } \\
\text { S }\end{array}$} & \multicolumn{2}{|c|}{ I Gravilà ridotla secondo } & \multicolumn{2}{|c|}{ Anomalie } \\
\hline & $\begin{array}{c}\text { IIAYHORD } \\
\mathrm{g}^{\prime} \mathrm{e}^{\prime}\end{array}$ & $\mid \begin{array}{c}\text { HEISKANEN } \\
8 c^{\prime \prime}\end{array}$ & $\ddot{\prime}^{\prime}{ }^{\prime}-\gamma$ & $\because "$ \\
\hline Rucial di Papa & $\begin{array}{c}\text { gal } \\
+980.117\end{array}$ & $\begin{array}{c}\text { inal } \\
+980.125\end{array}$ & $\begin{array}{c}\text { Ial } \\
-+0.079\end{array}$ & $\begin{array}{c}\text { gral } \\
+0.087\end{array}$ \\
\hline Monte Cavo & +980.125 & +980.135 & +0.088 & $\because 0,098$ \\
\hline Ariceria & +980.383 & $+980.38 \%$ & -0.0 .19 & +0.05 .1 \\
\hline Castel Gandolfo & +980.413 & $\div 980.411$ & +0.075 & +0.078 \\
\hline Miarino & +980.102 & +980.105 & +0.001 & $+0.06 \vec{i}$ \\
\hline Frascali & $+980,383$ & +980.385 & $\therefore 0,0.11$ & $\cdots 0.01 .13$ \\
\hline Ciampino & $+980,108$ & +980.116 & $+0.06 \pi$ & $\therefore 0.075$ \\
\hline Fratlocelie & +980.396 & +980.396 & +0.058 & $\div 0.058$ \\
\hline Vemi & $+980,-103$ & +980.108 & +0.069 & $+0.0 \div .1$ \\
\hline Velletri & +980.380 & +980.381 & $+0.0-19$ & +0.050 \\
\hline Montecompatri & +980.398 & +980.101 & $\div 0.056$ & +0.059 \\
\hline Rocea Priora & +980.397 & +980.397 & +0.1157 & $\because 0.057$ \\
\hline Calpo di Bove & +980.393 & +980.384 & +0.038 & +0.039 \\
\hline Scuola Media Agraria & +980.370 & +980.371 & +0.025 & +0.029 \\
\hline IV Niglio & +980.371 & $\div 980.37 .1$ & $\div 0.026$ & -0.1129 \\
\hline Catslel di Leva & +980.361 & +980.362 & +0.023 & +0.02 .1 \\
\hline Scuolal D. G. Bassi & +980.360 & +980.362 & +0.019 & $\dashv-0.021$ \\
\hline Mandriolia & +980.377 & +980.379 & +0.039 & +0.01 .11 \\
\hline Lanumio & +980.358 & +980.360 & +0.1028 & $\div-0.030$ \\
\hline Nistri & +980.370 & $+980,372$ & +0.023 & +0.025 \\
\hline Patronat & +980.375 & $\therefore-180.376$ & $\therefore-0.11 .10$ & +0.01 .11 \\
\hline Cerchina & +980.356 & +980.359 & +0.025 & +0.028 \\
\hline S. Cesareo & +980.373 & $+980.37+1$ & +0.030 & +0.031 \\
\hline Panlano & +980.357 & +980.358 & +0.010 & +0.011 \\
\hline Jorre Nova & +980.362 & +980.362 & +0.015 & -0.015 \\
\hline Cisterna & +980.370 & +980.379 & $\div 0.056$ & +0.057 \\
\hline Cori & +980.130 & +980.135 & +0.1013 & +0.108 \\
\hline Giulianello & +980.375 & +980.376 & +0.0111 & +0.045 \\
\hline Lariano & +980.389 & $+980,390$ & +0.05 .1 & $\div 0.055$ \\
\hline Valmontone & +980.359 & +980.360 & +0.020 & +0.021 \\
\hline Palestrinal & $+980,393$ & $+980,399$ & $+0,0.18$ & +0.05 .1 \\
\hline Gallicano & +980.373 & +980.374 & $+0,025$ & +0.026 \\
\hline Tenuta Granaracoio & +980.371 & $+980,372$ & +0.019 & $\therefore 0.020$ \\
\hline Lunghezza & +980.362 & +980.363 & $+0,010$ & +0.011 \\
\hline Sellecimini & $+980,367$ & +980.367 & $+9) .013$ & +0.013 \\
\hline
\end{tabular}


a--unlo rome den-ila di calcolo quella media di 2.2 delle roece esitenti nella regione: le anomalie trovale vamo interpretate appmo come dovute a rore di den-ila diver-a fa guella media as-unla.

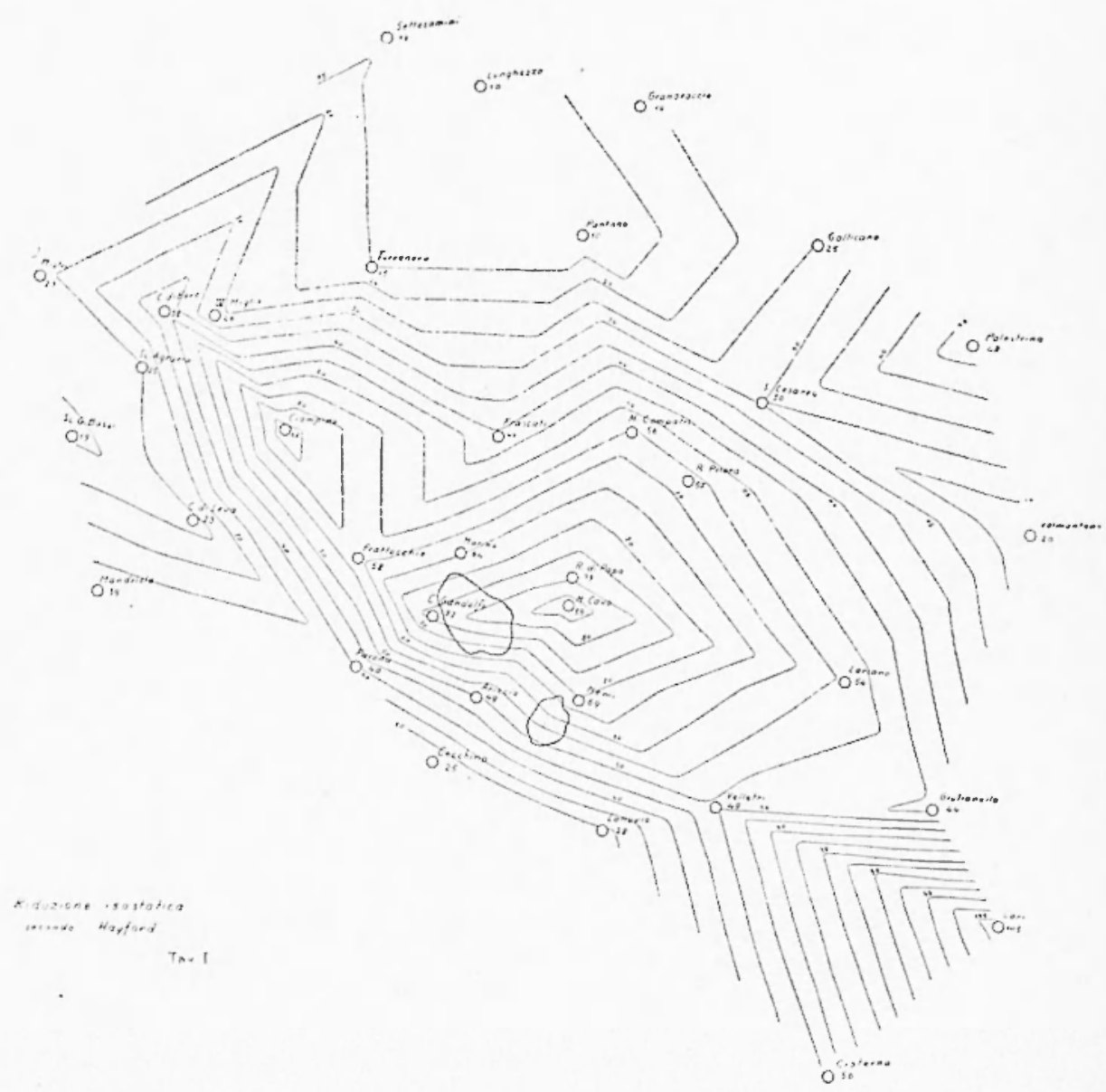

Dalla labella III rileviamo che $\mathrm{i}$ valori di gy ridonli seguendo il peocedimento di Bouguts differiscono da quelli di Fare, di Harromb

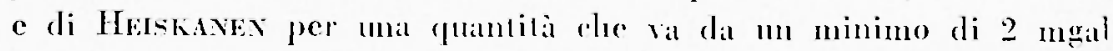
ad 111 matsimo di 52 mgal.

Prima di passare alla discusione dei risulati riportati vogliamo riasmmere brevemente i dali geologici della regione in modo che sia poj possibile fare un parallelo tra i risultati otlenuti e quelli che si 
sarchbero dowuti atlendere in base alle sole caratteri-tiche geologidhe.

La regione in esame, sede del Vulcano Laziale. puó, lopograficalmente, considerari mediamente aceidentala: quote masime- -li lom metri, per una parte, e la rampagna romana rhe, in vari punti, riname notevolmente sotto i lon metri. Queste condizioni -ono tali da es-ere lisentite nei risultali delle osiervazioni.

Uno sguardo ia pure rapido, alla regione, rivela l'esi-tenza di un grande cratere, primitivo (detto dell'Arlemi-io o Tureolano), del diat metro esterno di una ventina di chilometri, ed interno di una decina. interrotto nellat parte oves e sud-sud-ovest rispettivamente dati rateri di Castel Gandolfo (Lağo di flbano) e di Nemi (Latgo omonimo): internamente a questo $i$ nota poi un aratere più pireolo (detto di Monte Albano o Monte Caco) rle raggiunge i 950 m rireat.

Più oltre, a sud-ovest, si trova la valle di Ariccia anchensal con la rallateristion forma di cratere.

Tulta la regione circostante inolue è cosparsa di numerosi a apparechi aventizi ", alemi dei quali, lungogo le vie Anagrana ed Appia. ragrgiungono Romal.

I terreni che i riseontrano in questa regione sono enenzialmente vulcanici e comprendono tufi da litoidi a terrosi. pozzolane, lave leuriliche: essi hamno una potenzat variabile da aleune decine ad aleme rentinatia di melri e powgiano direttamente sopra la potente formatzione delle argille e sabbie plioceniche.

Per fuanto riguarda le loro densili si può assumere per i lufi e le pozzolane un valore medio di 2.2 , per le lave un valore medie di 2.7. per le sabbie e argille un valore medio di 2.

Rirordiamo infine rhe la regione in came prima fortemente vulcanical, sebhene ormai dentinata ad un progressivo areesamento. i. luttavia, come dimostra la storia recente ancora al giomo d’ogegi, sottopo-iat at una notevole attivila sismica.

Solo nellultimo secolo -i wno infatti rerifuati foltre ad altre seose minori): due terremoti di eui uno rovinoso, con epicentro a Frascati; tre, di eni due fortimimi, con epicentro ad Albano: due ron epiecnte ad Ariccia; uno con epicentro a Genzano ed uno rovinowo con picentro presso Nemi: le profondita ipocentriche sono comprese fra un minimo di $3 \mathrm{~km}$ ed un mar-imo di $10 \mathrm{~km}$ e pue-lo permette, dato rhe le caratleristiche di tali moti ateruano anzaltro che trattasi di teremoli di crollo, di avere un elemento indiretlo. soblene non precioo, cirea la profondita del batcino magnation. 


\section{TAPELIA III}

Anomalic secondo Bonguer

\begin{tabular}{|c|c|c|}
\hline $\begin{array}{c}\text { Sarzioni } \\
S\end{array}$ & $\begin{array}{l}\text { Gravità ridotta secondo } \\
\text { la riduzione totale } \\
\text { di Botcien } \\
\text { o }=2.2\end{array}$ & $\begin{array}{l}\text { Anomalie } \\
\qquad \mathfrak{c}-\dddot{i}\end{array}$ \\
\hline Roceal rli Paupa & 980.393 & $\therefore 0.1155$ \\
\hline Nonle Caro & 379 & 12 \\
\hline Aricria & 376 & 52 \\
\hline Calselgandolfo & .111 & 75 \\
\hline Miarino & 108 & 70 \\
\hline Fra-cali & 399 & 57 \\
\hline Ciampino & 434 & 93 \\
\hline Fiallonerhis. & $\$ 15$ & 77 \\
\hline Vemi & 395 & 61 \\
\hline Velletri & 393 & 62 \\
\hline Monlecompatiti & 396 & $5-1$ \\
\hline Roceal Priora & $37-1$ & $3 \cdot 1$ \\
\hline Capo di Bove & $-10 \bar{T}$ & 62 \\
\hline Scuola Media Agraria & $39 \cdot 1$ & 19 \\
\hline IV Miglio & 395 & 50 \\
\hline Cantel di leval & 380 & 12 \\
\hline Sruola G. Basi & 383 & 42 \\
\hline Mandriola & 397 & 59 \\
\hline I anuvio & 373 & 13 \\
\hline$N i=1 \mathrm{ri}$ & 396 & 19 \\
\hline Parona & 396 & 61 \\
\hline Cecohina & 374 & 13 \\
\hline S. Ciesareo & 392 & 49 \\
\hline Pantano & 398 & 51 \\
\hline Torre Foya & 389 & 12 \\
\hline Cislerna & $10 \%$ & 36 \\
\hline Cori & 135 & 108 \\
\hline Giulianello & 102 & 71 \\
\hline Lariano & 105 & 70 \\
\hline Valmonlone & 303 & 44 \\
\hline Pallestrina & 402 & $5 \pi$ \\
\hline Gallicano & 398 & 50 \\
\hline Torre Granaraceio & 411 & 59 \\
\hline I,unghezzal & 402 & 50 \\
\hline Seltecamini & $10 \overline{5}$ & 51 \\
\hline
\end{tabular}


Interprelazione dei risultali.

Per passare dai dali analiliei delle labelle 1 . Il e III alla loro interprelazione alla lue delle precedenti ronsiderazioni sula naturat peologica della regione. abhiamo traceiato nelle las. I-IV le curve i-omomale oflenule serondo le diverse leorie.

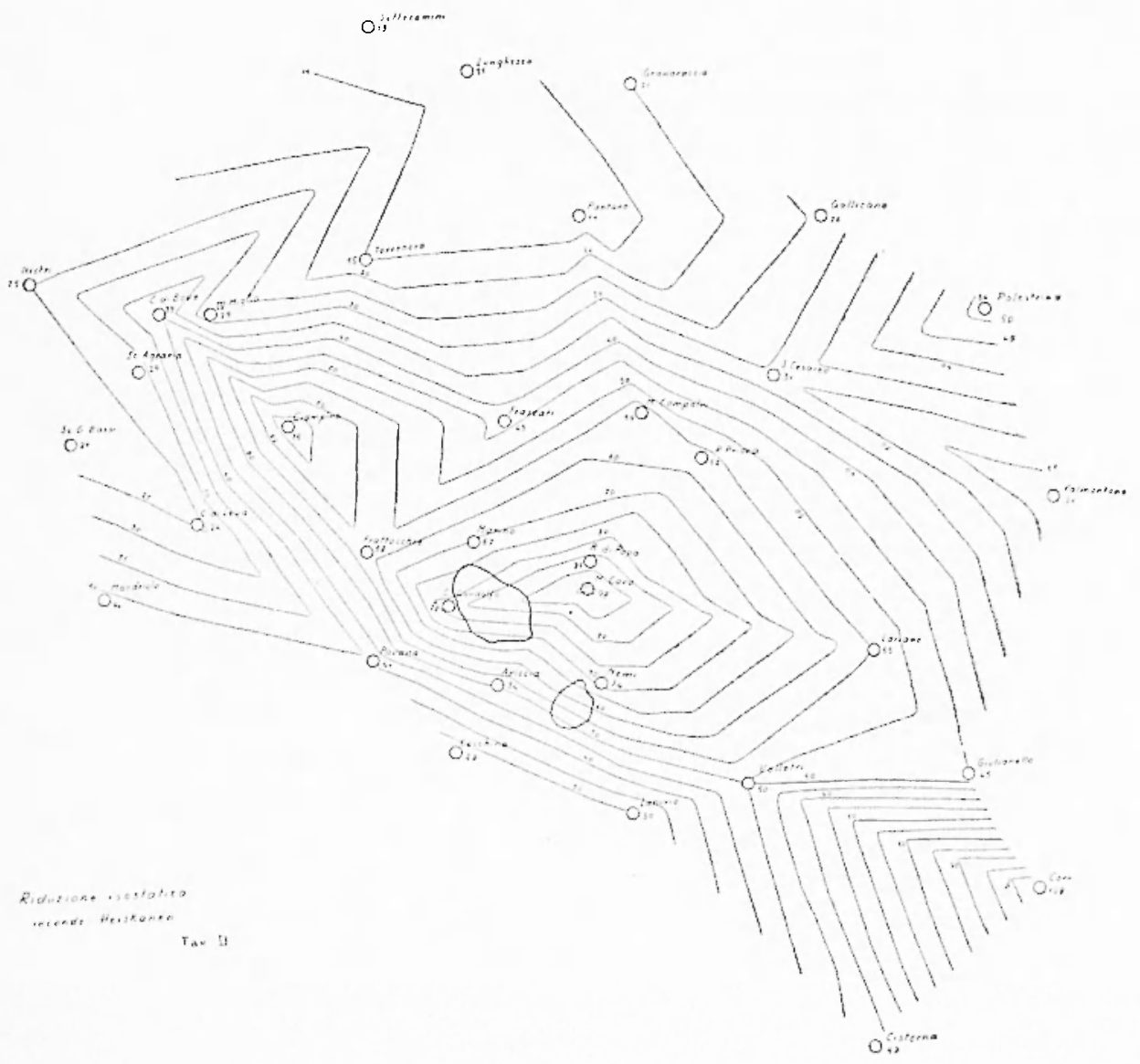

Le lav. 1 a II riportano le isoanomale di Homoro e Thokinex.

L'anctamento delle ivomomale riportale in delle tarole, mollo -imili tra di loro, aceusa un masimo nella zona growolanamente delinilante il ralere Tuscolano.

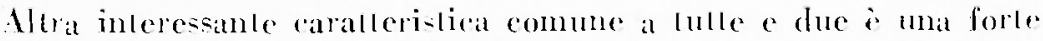
aredenza, positiva, nella regione di Ciampino (acroporto). Fe questa una collina che ragrgiunge la masuma quota proprio in detla localia. 
La lavola III i quella rle riporla le i-oamomale ollemule secondo Fare. Anche in reue-ta rappre-entazione ei trosiano dasanti ad anomalie con due regioni di ma-simo comi-pondenti prewo a poeo a quella delle anomalie i-ostatiehe preecelenti.

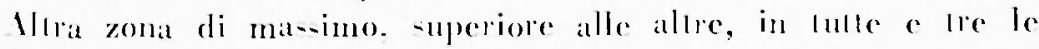
tavole. ¿q quella di Corri.

Ina resione di minimo -i delinea infune. nelle lavole $T$ a II nelle Loralila Sellecamini. Torrenoca. Pantano. Langhezza.

Nella lasola 111 detla regione si estende al II Migho.

Lat lav. IV riportat da ulimo le i=o:anomale oltenule secondo Bod berr. Ia media delle anomalie lrovale, fulle positive, i ri-ultala di ז... m mạal.

Ri-pello a fue-lo -i notano. nella lavolat:

1) una regione di minimo in rorrispondenzat al ralere dell'trli'misio: lat eonformazione planimetriea delle isoanomale is allumgatat dirella da S-O veroo Y-E. qua-i ad alle-tate ehe in lempi remoli i prelevamenti del matgma elano falli. forae. eerentidamente (in corfi-pondenzia di Rosen Priora). poi disl rono di M. Cano:

2) Hna regione di masimo (sempre rispello alla media di $5,7,7$ moal) in corrispondenza di Ciampino ed in areordo ron le Tarole precedendi: le aurve rag̣giungono. a V-O, Capo di Bowe, includendo lat colala di lava omonima: a S.E. le Frallocelie:

3) infine la regione di Corr fper la puale peró non ahbiamo punti eireo-lanti a di-proizione) in cui si ri-contra ma forle anomalia po-iliva. anomalia ricontrala gria ancherea nelle lavole I. II a 111.

Confrontando i ri-ulati otlenuti apportando le riduzioni secondo i fulllro melodi riporlali s grumge perlanto alle seguenti ronelusioni :

1) Con lulli i melodi di riduzione ci melle in vi-lat leaslenza di una anomalia po-iliva senerale delia requone. Tale anomalia po-ilisa trosa ma spiegazione immediala nella peolowia nota e prenatmibile dellat regione in quanlo e.ea pui interpetalsi come dovila alla esi-lenza della massa del bateino matrmatico ehe hat alimentalo l'alli-

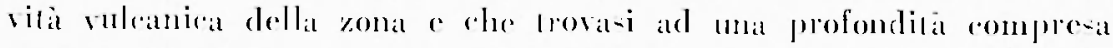
fra 3 . $10 \mathrm{~km}$.

2) Con tulli i melodi le mi-ure di wratila demolano in pros-imila di Ciampino e nella regione di Cori la presenzal di alee aventi mal forle eredenza di destia ri-pelio all'anomalia posiliva renerale della regione. Trala-eiando di parlate della regione di Cori, per la

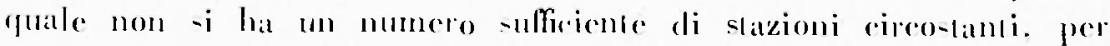
quanto riguarda il rilievo enpoliforme in rerri-pondenza di Ciampino 


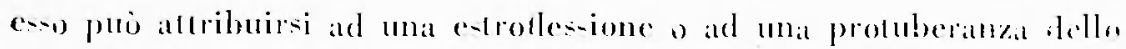
-leso bacino magmatico principale: ad es-at presumibilmente -i puis ricollegare anche leffu-ione laviea di Calpo di Bove la quale -arehbe

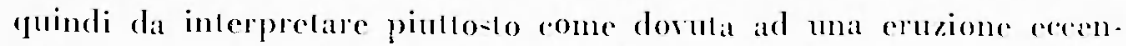
Irica, come altri elementi di superficie e come l'analogia ron alta lave della regione indueono a ritenere probubile.

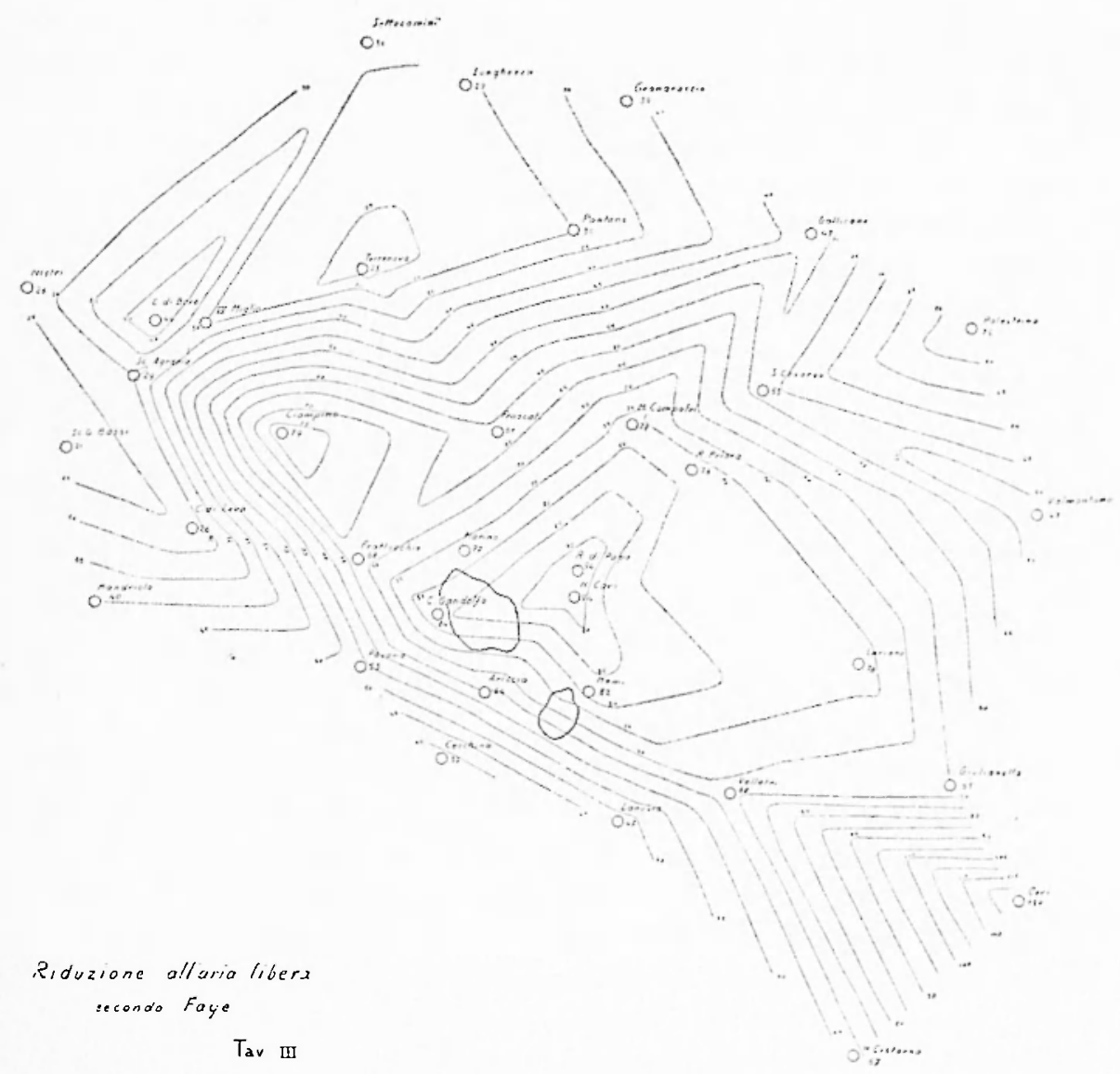

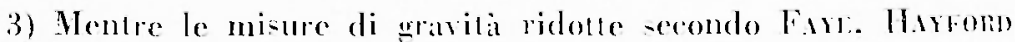
e Hotskaves indicano sotto l'appatrato del centro craterico di Nham le-istenza di ma forte cenedenza di demsiti rispello al valore medin dellanomalia positiva della regrione. le secse misure ridotle sceondo Botigcer, indicano invere un area di depresione relativa all andamento gencrale. 
Vel ra-o della riduzione all'aria libera di Fuse lale di-erepanza

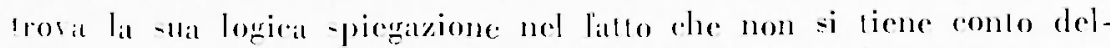

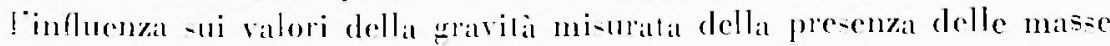
...iente sopra il lisello del watre e fuindi i valori riportali ri-nlamo

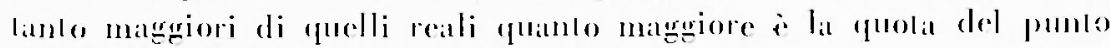
di stazione -nt livello del mare.

Nel raso delle siduzioni i-ostatiche i lapote-i amme-al che lar

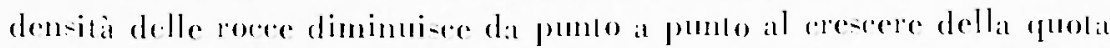

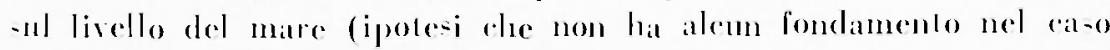

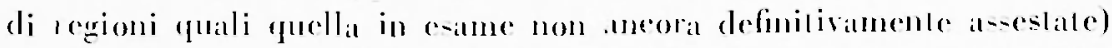
de eondue a sollovalulare lazione sulle mi-ure della gravila delle

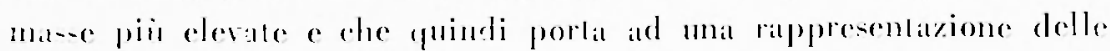
i-oanomale rhe ri-enle nolevolmenle, sebbene in minor mi-ura ahe nel melodo Fine, dell'andamento topogratico del terreno.

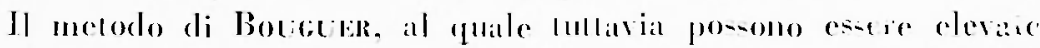

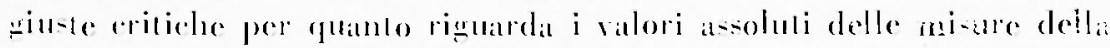

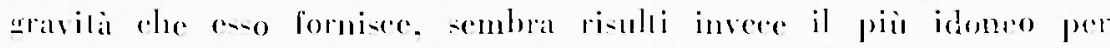
mellere in aidenzal le differenze dellat densila delle roene relalis ia

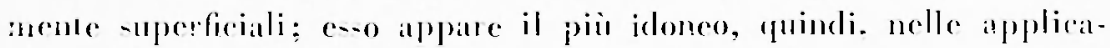
rioni dellat gravimelria ai problemi di greologaia lociale e li geolonia mincrirria.

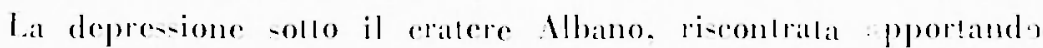

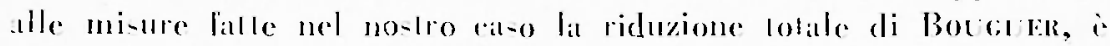
infiali pienamente gimslifuabile -ulla bate della geologia della regione: ear pui infalli ancriversi allo -ruolamenlo della parlie -uperiore del batcino magmatiog, suotamento rhe ha originalo fad mal profon-

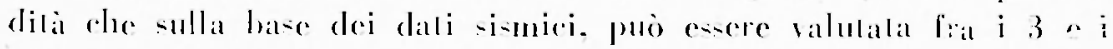
$10 \mathrm{~km})$ ma abbistamza esle-al zonal di dem-ila in ogni raso molto minore della media cireo-lanle. A ronferma di fuesta affermazione rosliamo fin vedere infalli, con ralcoli puramente indiralisi fperela troppo -emplificalivi) che le defieienze di mat mat-si probabilmente.

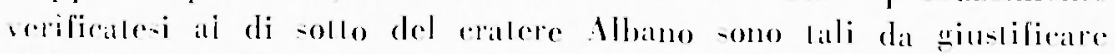
lubicazione e loordine di grandezza delle anomalie riecoulralle nellapplieare la correzione di Botribre.

Ricordiamo anzilullo ehe ma valustzione volumetrien dei malle. biali fuori w-eili dai diserei reraleri del Vuleano Laziale fe rhe lutiavia non liene conlo ni dei prodolli gat-oni -prigionalisi. ne dei prodolli sucecesirmente asportali dalle ero-ioni, ere.) is salia liatla dial Sabatine o fic-ala a poeo meno di $190 \mathrm{~km}^{3}$. Arrolondando a 200 que-10 valore a suppotendo la loro den-ila pari a $d=2.2$ la masa 
risultante sari pari a $4.10 .10^{9}$ t. Strpponendo per somplifieare il valcolo, whe tulla questa ma-ra fuori uarita da diver-i centri erullisi

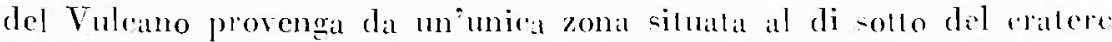
di M. Cavo e whe tale zona non sia stalat suce-ivamente neppure in pirte riempita da materiale collato dal tetto. il noto areatosi e ri-

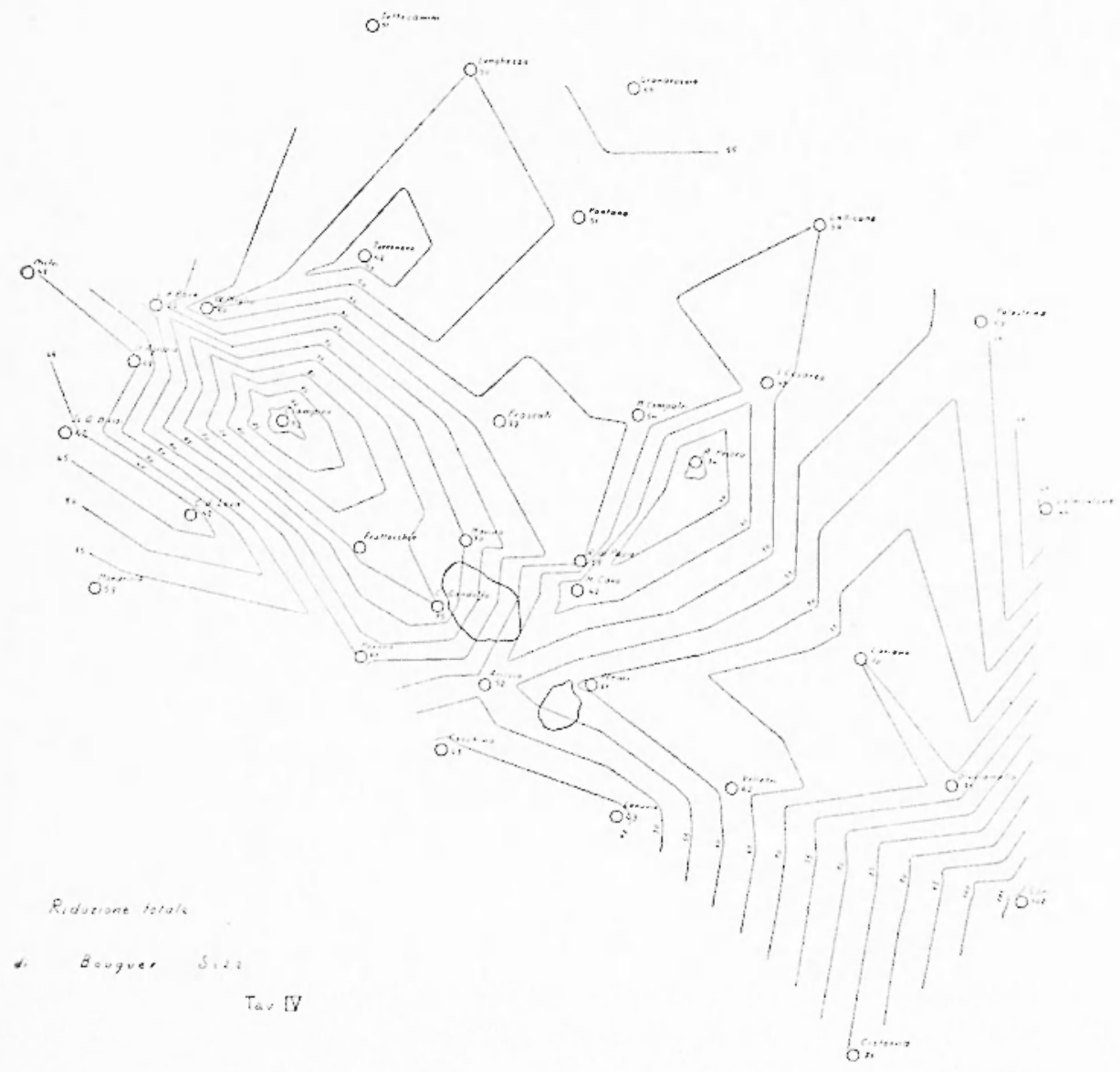

masio tale, supposto di forma ilindrica e alto $\mathrm{km} 3$. arrebbe una base circolare di raggio $u=3,9.5 \mathrm{~km}$.

L'effetto in superficie di un tale viroto, ralcolato ron lia nola e-presione

$$
F=2 ! \omega \tau \delta\left[H+a-y^{\prime} H^{*}+c^{2}\right]
$$

dove $\|=6673 \cdot 10^{-11}$ è la costante d'altrazione univesale, H la pro- 


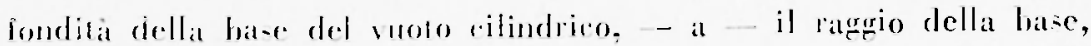
si-ulta di -37 maal nel ra-o che il dello woto e compreso frat la profoncila di $7 \mathrm{~km}$ c di $10 \mathrm{~km}$.

L'anomalia registrata a M. Cavo d di $42 \mathrm{mgal}$, diflecente, in meno. dal valore medio trovalo $(5 \pi, 7)$, per 15,7 mgal; l'anomalia regi- Hala invere a Rocea Priora, di 31 magl, differisce dalla media, di 23.7 mgal in menos.

Si vede dunque rome nono-tante le grosolane ipote-i fatle e nono-lante la incerteza dei dali impiegati, vi e un notevole areordo di frima approwimazione lra i dati calcolati e quelli ottenuti spe-

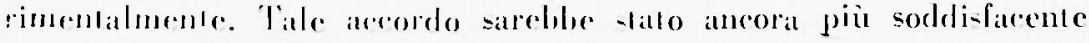

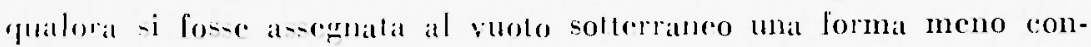
renlata (in particolare se grli if fo-e data una forma che per esempio

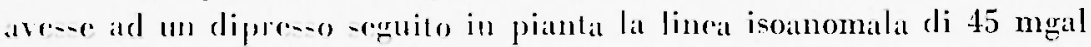
palatule per M. Calvo e Rocra Priolat, e qualora si fose temuto conto rler parte de

Roma - Istituto ali Goolisira Mincraria - giagno 194.

\section{RIASSUNTO}

Somo slali ripresi i risuluati de!lo ossertazioni statimetriche esesuile sui colli Albani a giò precedernemente pubblicuti; ad cssi sono late fatle le ridazioni secondo le diurese teorie a precisamente la ri-

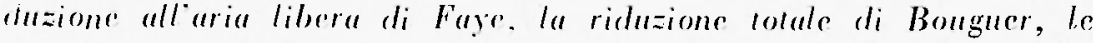
riduzioni isostatiche di Hayford-Bomeie a Ileislianon: si sono quindi tiportati sia in abblle che in grufiei i valori dolle anomalie gratimetriche oltermuse.

Sicopo del laworo i state duplices du una parte si e colula segaire la temdenza dei geodeli ifaliani a fare le ridnzioni isostaliche di lutle 16 slazioni gratimetriche misurate nel passe. dall'alura si è volmo sabilire in un case pratico a sopra un terreno geologicamente abbat- tama noto l'onsiti dolle differenze dri ralori o delle anomalie ri-

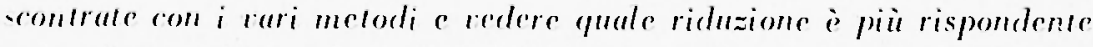
agli efgelli dello studio di problemi minerari interessanti profondiua ed cstemsioni limilate o zone non ancora completamente assestale.

Il confronto dei cari risullati permetle di stabilire che quelli se-

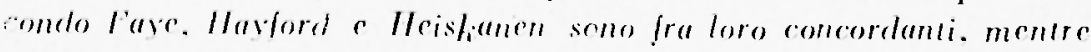
¿quelli sereondo Bouguer si discostano da essi. 


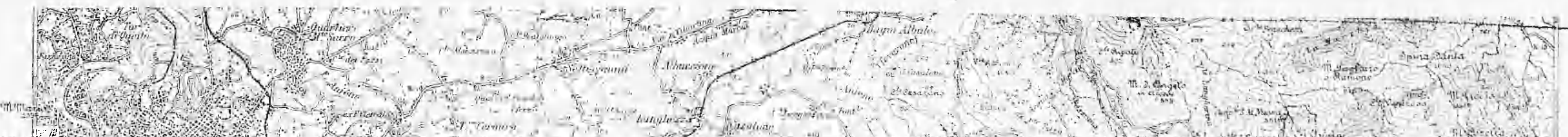

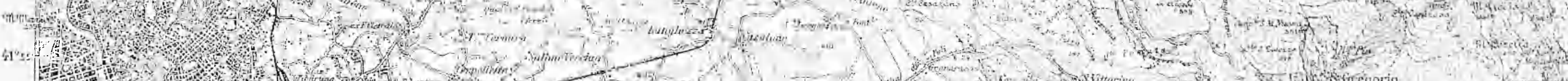

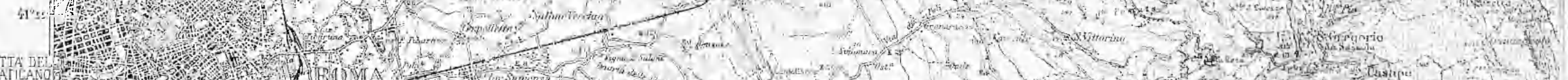

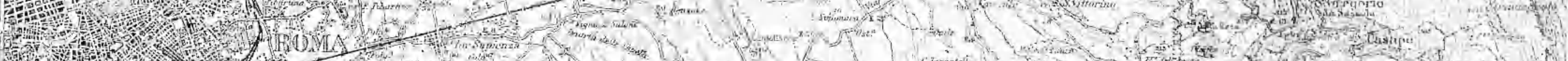

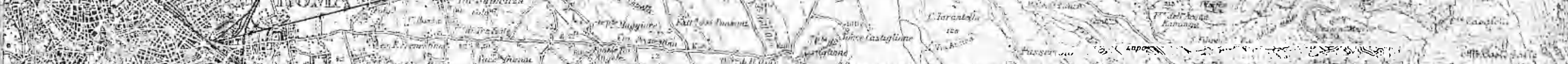

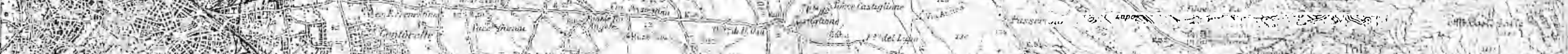

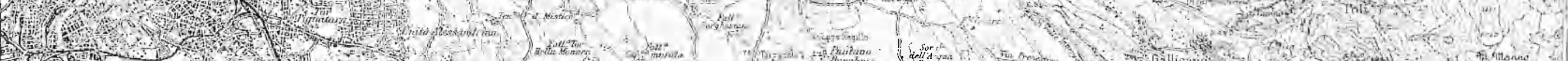

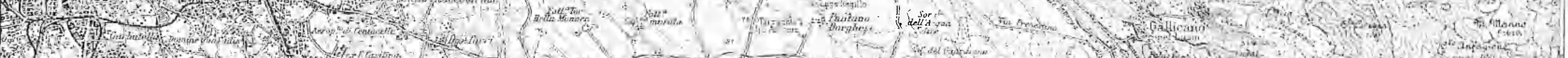

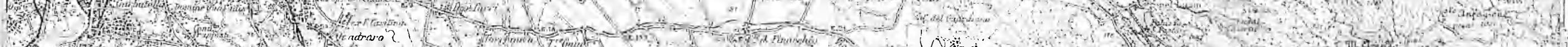

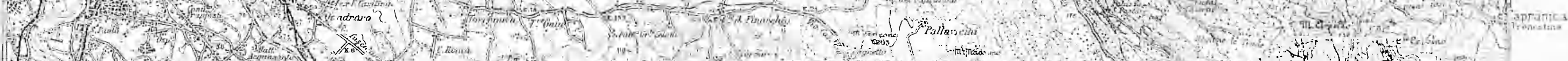

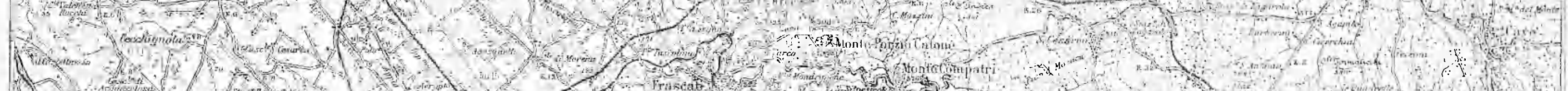

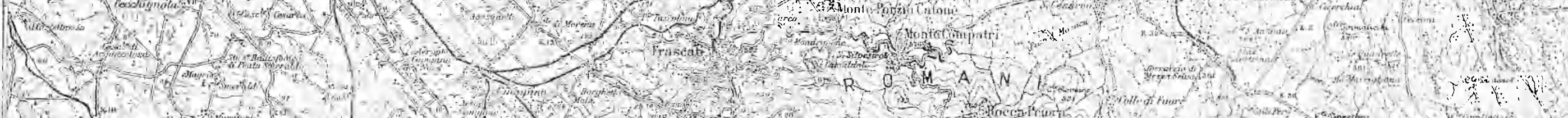

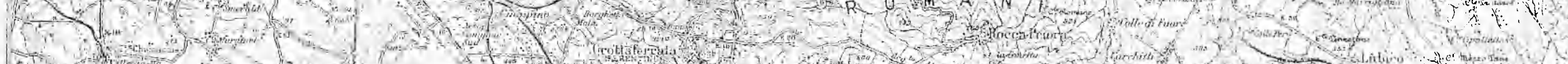

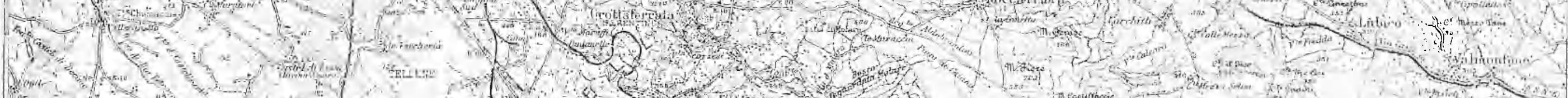
Xom

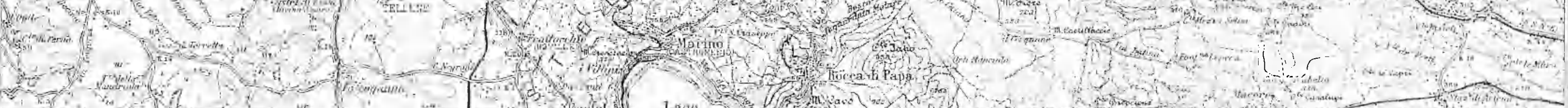

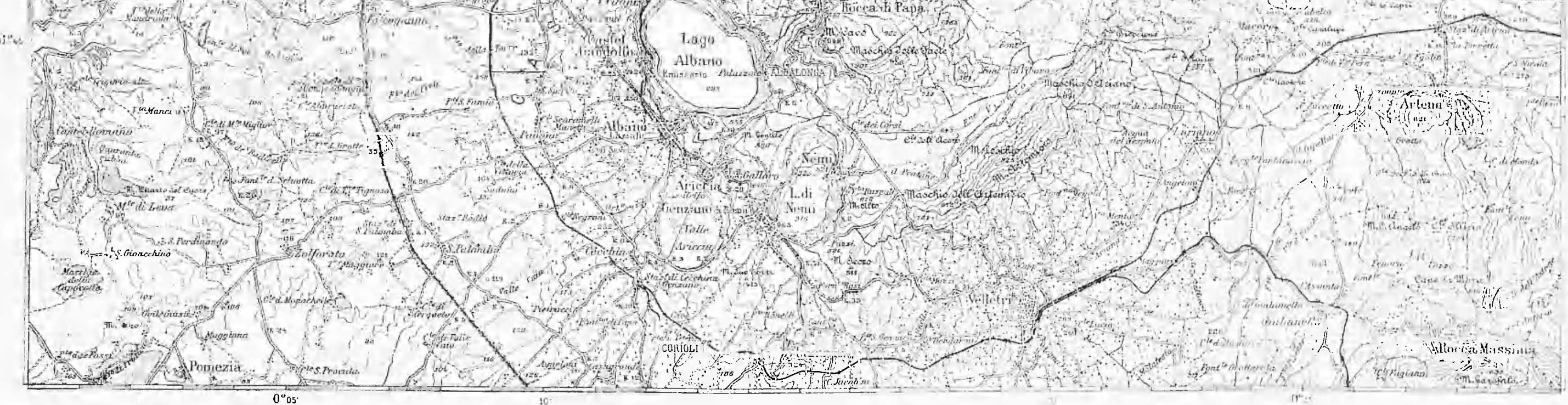


$1 /$ confronto con gli elementi noti dellu geototia tocate porterebb"

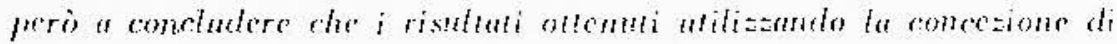

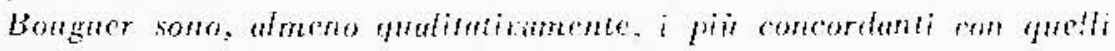
provedibili in base alle comaderusioni geologiche.

\section{BIBTIUGRAFIA}

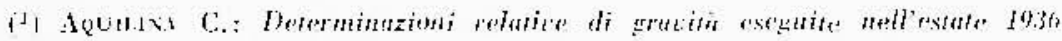

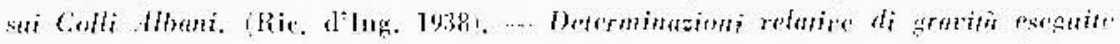

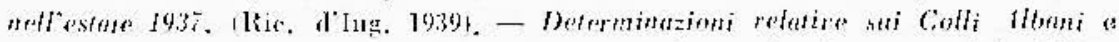

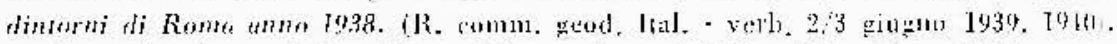

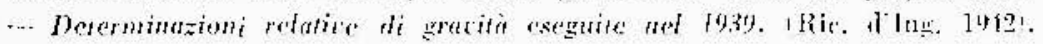

